\title{
Design of a radiation plate for load testing of the torsional transducers
}

\author{
Masahiko Takakusaki, Jun Satonobu, Yoshikazu Koike, Kentaro Nakamura, \\ and Sadayuki Ueha \\ Precision and Intelligence Laboratory, Tokyo Institute of Technology, \\ 4259 Nagatsuta, Midori-ku, Yokohama 226-8503 Japan
}

(Received 31 March 1998)

\begin{abstract}
This paper presents a design method of a radiation plate with high load resistance for load testing of the torsional transducers. The calculation method for the torsional load impedance of the radiation plate seen from the transducer is proposed considering the flexural vibration mode excited in the radiation plate. The theoretical study using this calculation method indicates that the use of the "stripe" flexural vibration mode has advantage over the use of the "lattice" mode in controlling and obtaining a high load resistance. Furthermore, by using a combination of changing the node number of the stripe vibration mode and changing the thickness of the radiation plate, the design of the wide radiation plate which achieves a high load resistance without changing the resonant frequency is attempted. In an experimental study, the measured torsional load resistance of the prototype radiation plate agrees with the calculated one, and the torsional load resistance of $4.7 \mathrm{Nms}$ was achieved with a $223 \mathrm{~mm} \times 50 \mathrm{~mm}$ radiation plate where the fifth stripe flexural vibration mode was excited. Load testing of the torsional transducer with the high torsional load was effectively conducted using the radiation plate designed by the proposed method.
\end{abstract}

Keywords : Torsional vibration, Transducer, Radiation plate, Load impedance

PACS number: 43. 35. $\mathrm{Yb}, 43$. 38. Fx

\section{INTRODUCTION}

The torsional vibrator has been developed to meet some high power ultrasonic applications including ultrasonic motors, ultrasonic welding, and ultrasonic machining systems. In some previous works, several characteristics of the torsional transducer such as the resonant frequency, the free motional admittance, the mechanical quality factor, and the torque factor have been investigated in detail to evaluate the performance. ${ }^{1-3)}$ These characteristics can be measured without loading the torsional transducer. The load characteristics such as the maximum output power and the maximum efficiency of the torsional transducer are also important. However, in order to measure the load characteristics of the torsional transducer, it is necessary to establish the methods for measuring the mechanical output power and for applying the torsional load on the output surface of the torsional transducer. Several methods to measure the mechanical output power for the longitudinal vibrations have been investigated ${ }^{4-6)}$ and they have been applied to the measurement of torsional output power. On the other hand, there have been few studies on the method to apply the torsional load on the transducer. Kaneko et al. have proposed the use of the radiation plate which rotates in water for this purpose. $^{7,8)}$ In this method, it is possible to change the load resistance easily by changing the immersed depth of the radiation plate, however, the value of the load has not been enough because the radiation plate used in the proposed system was small and the vibration mode of the radiation plate has not been 
taken into consideration. Furthermore, a method for calculating the torsional load impedance of the radiation plate has not been proposed yet.

The authors present the design of a radiation plate with high load resistance for load testing of the torsional transducers. An analytic expression for the torsional load impedance of the radiation plate vibrating in water is obtained considering the flexural vibration mode excited in the radiation plate. The theoretical investigation for the radiation plate indicates that using the stripe flexural vibration mode excited in the radiation plate has an advantage over the lattice mode in controlling and obtaining the high load resistance. In the experiments, the measured maximum load resistance of the prototype radiation plate agrees with the calculated one, and the load resistance of $4.7 \mathrm{Nms}$ was achieved with a $223 \mathrm{~mm} \times 50 \mathrm{~mm}$ radiation plate where the fifth stripe flexural vibration mode was excited. This value is about 100 times as large as the load resistance achieved in the previous work. The load test of the torsional transducer with the high torsional load was effectively conducted by using the radiation plate designed by the proposed method.

\section{CONFIGURATION OF THE MEASUREMENT SETUP AND THE VIBRATION MODE OF THE RADIATION PLATE}

Figure 1 shows the configuration of the measurement setup for load testing of the torsional transducer. The torsional transducer is connected with the horn and the radiation plate is connected to the tip of the horn. The horn has the flange located in

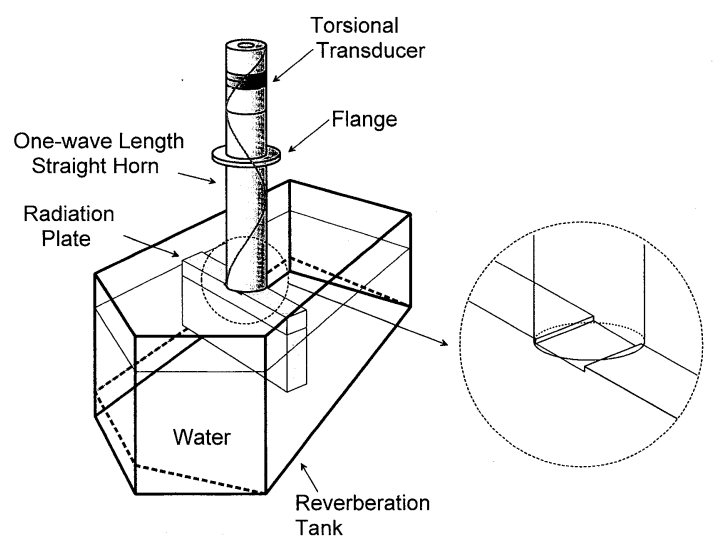

Fig. 1 Measurement setup the nodal plane of the torsional vibration mode and supports the vibration system. In order to obtain high load resistance, a large radiation plate where the flexural vibration mode is excited should be designed. Then, the radiation plate has a slit near the contact interface and the horn contacts the radiation plate at two small areas, identical to the outer circumference of the horn, so that the flexural vibration mode is excited in the radiation plate effectively. A reverberation tank is used to immerse the radiation plate with low influence of the standing wave field in water. Changing the immersed depth of the radiation plate in water does varying a load resistance.

The vibration system composed of the horn and the radiation plate should be designed to match the desired resonant frequency, identical to the resonant frequency of the transducer. The horn and the radiation plate can be designed separately, if each resonant frequency is identical to the resonant frequency of the transducer. The length of the horn, $l_{\mathrm{h}}$, whose torsional resonant frequency, $f_{\mathrm{h}}$, is simply determined from the following relation:

$$
l_{\mathrm{h}}=\frac{i}{2 f_{\mathrm{h}}} \sqrt{\frac{G}{\rho_{0}}}
$$

where $G, \rho_{0}$ and $i$ indicate the shear modulus, the density, and the mode number, respectively. The flexural vibration mode excited in the plate is represented as $(m, n)$ flexural vibration mode, where $m$ and $n$ indicate the number of nodal lines along

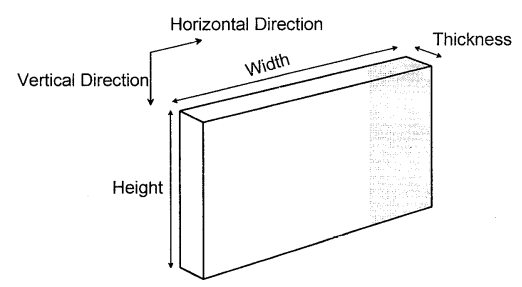

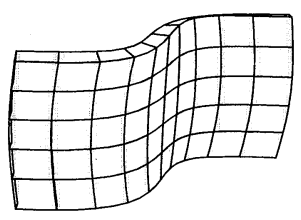

(a) $(3,0)$ Mode

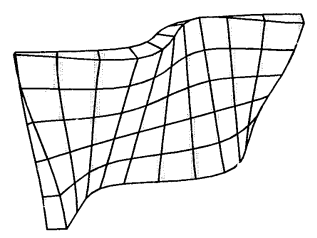

(b) $(3,1)$ Mode
Fig. 2 Examples of calculated flexural vibration modes excited in the radiation plate; (a), the stripe mode, (b) the lattice mode. 


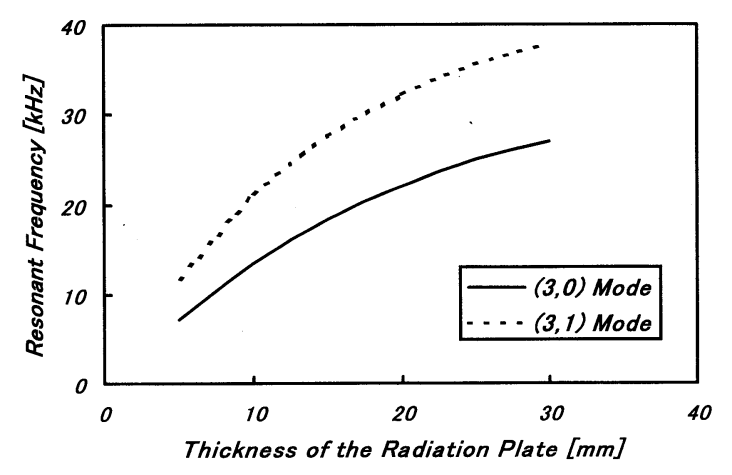

Fig. 3 Calculated resonant frequency as a function of the thickness of the $100 \mathrm{~mm} \times 50 \mathrm{~mm}$ radiation plate.

the horizontal direction and those along the vertical direction, respectively. The $(m, 0)$ or $(0, n)$ flexural vibration mode is generally called the stripe vibration mode, and the other is called the lattice vibration mode. The flexural vibration mode, which has an odd number of $m$, can be effectively excited because of the transformation from the torsional vibration mode in the horn to the flexural vibration mode in the radiation plate. Figure 2 shows the examples of the calculated flexural vibration mode excited in a $100 \mathrm{~mm} \times 50 \mathrm{~mm}$ radiation plate made of aluminum by the finite element method, ${ }^{9)}$ and Fig. 3 represents the resonant frequency of each vibration mode as a function of the thickness of the radiation plate. Two types of the vibration mode can be selectively excited by changing the thickness, and the resonant frequency of the radiation plate can be tuned by changing the thickness.

\section{CALCULATION METHOD FOR THE TORSIONAL LOAD IMPEDANCE OF THE RADIATION PLATE}

Figure 4 shows the schematic for explaining the calculation method for the torsional load impedance of the radiation plate. Let us assume that:

- The sound radiation from each face of the radiation plate does not propagate to the other face.

- The radiation from the side and the bottom faces of the radiation plate is small enough.

- The contacts with the horn are ideally made at two points equidistant away from the center of the radiation plate. The continuity of the circumferential velocity is maintained across the contact point, and the direction of the circumferential velocity at

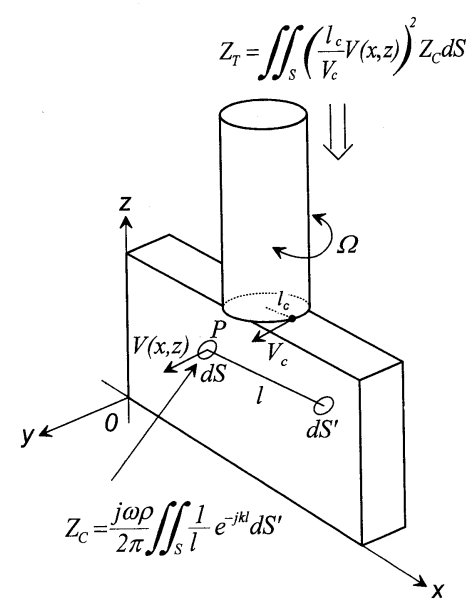

Fig. 4 Schematic for explaining the calculation of the torsional load impedance.

the contact point is identical to the direction of the flexural vibration of the radiation plate.

The radiation impedance at the point $P$ on the radiation plate is defined as the ratio of the pressure, $p$, to the velocity, $V(x, z)$, and is given by

$$
Z_{\mathrm{c}}=\frac{p}{V(x, z)}=\frac{j \omega \rho}{2 \pi} \iint_{S} \frac{1}{l} e^{-j k l} d S^{\prime}
$$

where $\omega, k$, and $\rho$ indicate the driving angular frequency, the wave number, and the density of the fluid, respectively. This impedance is transformed at the contact points, and seen as the torsional impedance from the horn, $Z_{\mathrm{t}}$, calculated from the following relation:

$$
Z_{\mathrm{t}}=\left(\frac{l_{\mathrm{c}}}{V_{\mathrm{c}}} V(x, z)\right)^{2} Z_{\mathrm{c}}
$$

where $l_{c}$ and $V_{c}$ indicate the distance from the center of the horn to the contact point and the circumferential velocity at the contact point, respectively. Finally, the total torsional load impedance of the radiation plate seen from the transducer is given by

$$
Z_{\mathrm{T}}=\iint_{S}\left(\frac{l_{\mathrm{c}}}{V_{\mathrm{c}}} V(x, z)\right)^{2} Z_{\mathrm{c}} d S
$$

and the real part of this equation represents the load resistance of the radiation plate.

\section{METHOD FOR DESIGNING THE RADIATION PLATE WITH HIGH LOAD RESISTANCE}

First, let us consider the effective flexural vibration mode of the radiation plate to obtain high load resistance. Figure 5 shows the maximum load 


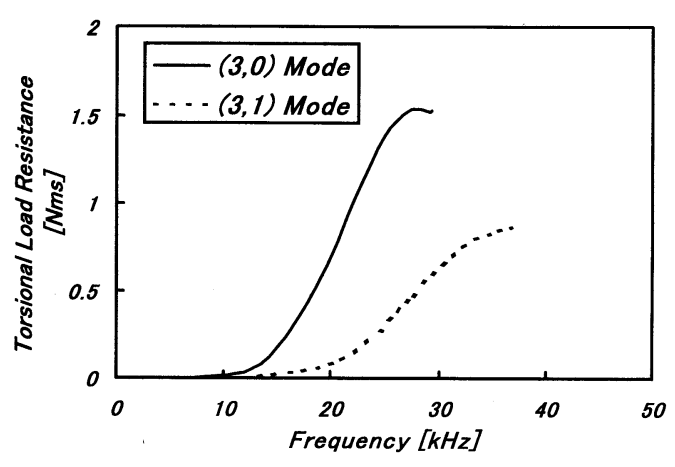

Fig. 5 Calculated maximum load resistance as a function of the driving frequency.

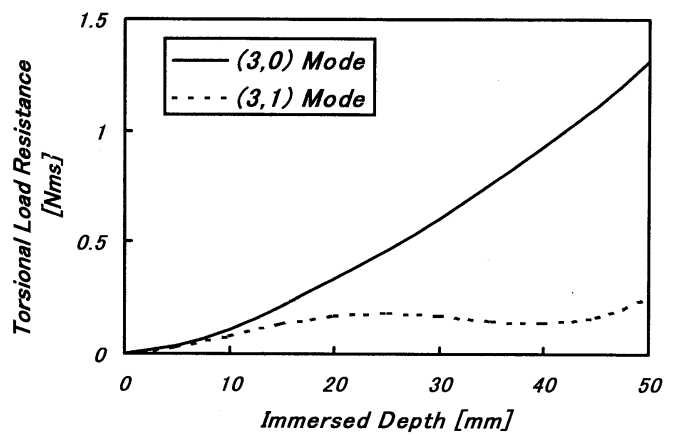

Fig. 6 Calculated load resistance as a function of the immersed depth in water.

resistance of the $100 \mathrm{~mm} \times 50 \mathrm{~mm}$ radiation plate from relation (4) as a function of the driving frequency where the immersed depth is assumed to be $50 \mathrm{~mm}$; identical to the height of the radiation plate. The thickness of the radiation plate was changed to match the desired resonant frequency; identical to the resonant frequency of the transducer, and the vibration mode was calculated by FEM for each driving frequency. The $(3,0)$ stripe vibration mode and the $(3,1)$ lattice vibration mode can be excited around the driving frequency from $20 \mathrm{kHz}$ to 30 $\mathrm{kHz}$, which is the resonant frequency of the torsional transducer used in the general torsional vibration applications. The load resistance of the stripe vibration mode is higher than that of the lattice vibration mode. Figure 6 shows the calculated load resistance as a function of the immersed depth of the radiation plate. The driving frequency is assumed to be $24 \mathrm{kHz}$; identical to the resonant frequency of the flexural vibration mode of the

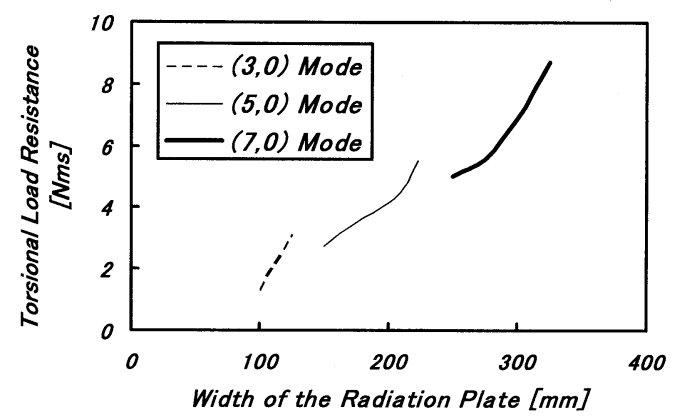

(a)

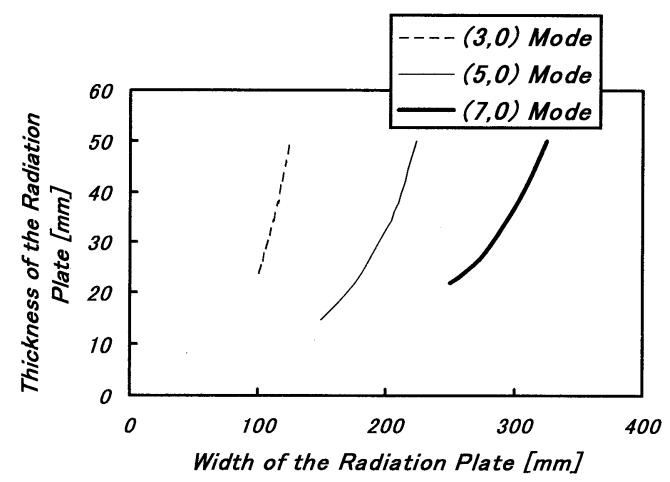

(b)

Fig. 7 Design chart for the radiation plate.

radiation plate. The radiation from the part of the plate in air is neglected for simplicity. The load resistance of the stripe vibration mode increases simply, while the load resistance of the lattice vibration mode varies complicatedly because of the nodal line along the vertical direction. Then, using the stripe vibration mode should be recommended for controllability of the load high load resistance.

Next, let us discuss the method to design a large radiation plate with high load resistance. In general, the resonant frequency of the radiation plate depend on its size, then, the method to design the large radiation plate without changing the resonant frequency of the stripe vibration mode is necessary. For this purpose, here, the design of the horizontally wide radiation plate which achieve high load resistance without changing the resonant frequency is attempted by the combination of changing the node number of the stripe vibration mode and changing 
the thickness of the radiation plate. Figure 7 represents the design chart for the maximum load resistance of the radiation plate of $50 \mathrm{~mm}$ in height as a function of the width, and the thickness of the radiation plate as a function of the width. The target resonant frequency is assumed to be $24.3 \mathrm{kHz}$. The radiation plate with the load resistance less than 9 $\mathrm{Nms}$ can be designed from this chart. For example, if the torsional resistance of $3 \mathrm{Nms}$ is needed, the radiation plate $125 \mathrm{~mm}$ in width for the $(3,0)$ flexural vibration mode can be chosen from the chart (a) and the thickness of the radiation plate can be determined from the chart (b) to be $50 \mathrm{~mm}$. The other choice is using the radiation plate $158 \mathrm{~mm}$ in width and $17 \mathrm{~mm}$ in thickness for the $(5,0)$ flexural vibration mode. The larger radiation plate for other frequency can be designed similarly.

\section{EXPERIMENTS}

\subsection{Measurement of the Load Resistance}

When the vibration system of the measurement setup is driven at its resonance, the equivalent electrical circuit model for the torsional vibration is introduced as shown in Fig. 8. $^{10}$ The clamped capacitance of the PZT and the dielectric loss are denoted by $C_{\mathrm{d}}$ and $R_{\mathrm{d}}$ respectively. The load resistance of the radiation plate, $r_{\mathrm{a}}$, is determined by measuring the angular velocity at the end of the horn, $\Omega$, the input voltage, $V$, the torque factor of the transducer, $A$, and the mechanical resistance of the overall vibration system, $r_{\mathrm{m}}$, from the following relation :

$$
r_{\mathrm{a}}=\frac{A V-r_{\mathrm{m}} \Omega}{\Omega} .
$$

The torque factor, $A$, represents the maximum output torque per unit voltage applied to the electrical port and can be approximately determined from the relation between the input current and the angular velocity without loading. ${ }^{11)}$ The mechanical resis-

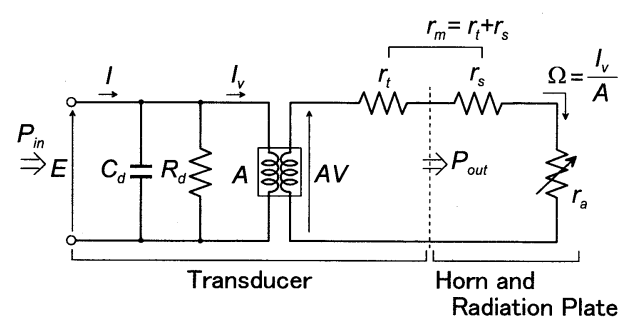

Fig. 8 Equivalent electrical circuit model of the measurement setup. tance, $r_{\mathrm{m}}$, is represented by the summation of the mechanical resistance of the transducer, $r_{\mathrm{t}}$, and the mechanical resistance of the vibration system composed of the horn and the radiation plate, $r_{\mathrm{s}}$, and is also determined by using the equivalent electrical circuit model.

The designed configuration and the dimensions of the prototype radiation plate and the dimensions of the reverberation tank used in the experiments are shown in Fig. 9. Figures 9(a) and 9(b) were designed for the excitation of the $(3,0)$ mode and the $(5,0)$ mode, respectively. The radiation plate and the horn were machined from a single aluminum plate so that the boundary area between the radiation plate and the horn was continuous. The resonant frequency of each prototype is $24.3 \mathrm{kHz}$, identical to the resonant frequency of the transducer. The measured load resistance of each prototype radiation plate as a function of the immersed depth is shown in Fig. 10. The measured maximum load resistance agreed well with the calculated one, and the maximum load resistance of the $(5,0)$ mode radiation plate was about 100 times as large as the previous work. ${ }^{7,8)}$ However, the experimentally determined value was slightly different from the calculated one, especially as the immersed depth decreased in the $(5,0)$ mode radiation plate. The authors speculate the radiation from the side and bottom faces affects the measured values, but further examination is necessary to confirm this explanation.

\subsection{Load Testing of the Transducer}

Let us show the examples of load testing of the torsional transducer. The simple method to measure the output mechanical power using the watt meter is introduced in order to conduct load testing of the transducer. ${ }^{4)}$ The output power from the transducer, $P_{\text {out }}$, is given by following equation ;

$$
P_{\text {out }}=P_{\text {in }}-P_{\mathrm{d}}-P_{\mathrm{t}},
$$

where $P_{\mathrm{in}}, P_{\mathrm{d}}$, and $P_{\mathrm{t}}$ indicate the input electric power, the dielectric loss of the PZT disk, and the mechanical loss consumed in the transducer, respectively (refer to Fig. 8). The input electric power, $P_{\text {in, }}$ can be measured by using the wattmeter. If the dielectric loss, $R_{\mathrm{d}}$, and the mechanical resistance of the transducer, $r_{\mathrm{t}}$, are known, the dielectric loss, $P_{\mathrm{d}}$, can be determined from the following relation by measuring the applied voltage, $V$ : 


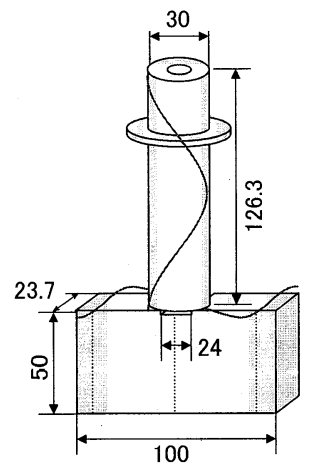

(a) $(3,0)$ Mode Plate

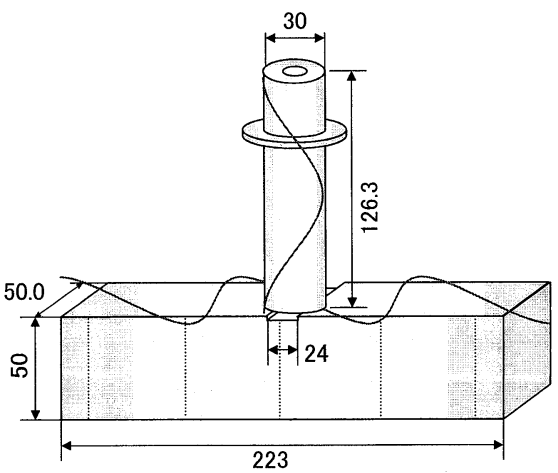

(b) $(5,0)$ Mode Plate

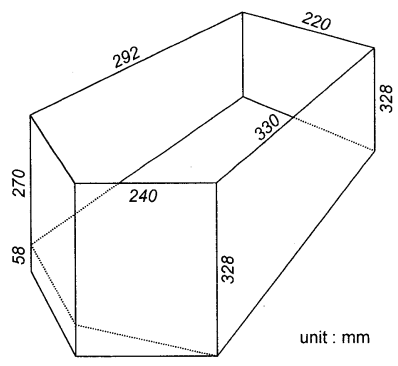

(c) Reverberation Tank

Fig. 9 Dimensions of the prototype radiation plate and the reverberation tank.

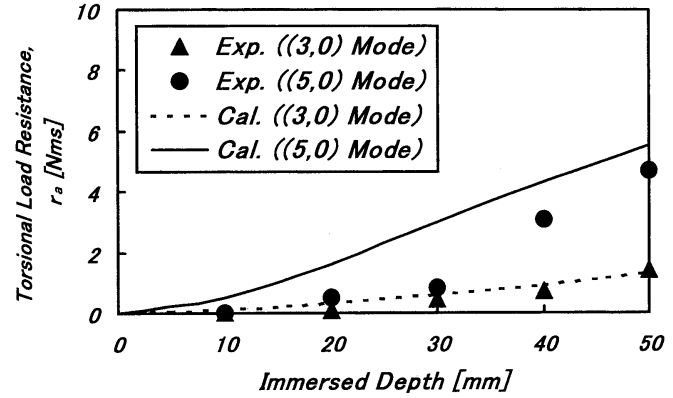

Fig. 10 Measured load resistance of the prototype as a function of the immersed depth.

$$
P_{\mathrm{d}}=\frac{V^{2}}{R_{\mathrm{d}}},
$$

and the mechanical loss in the transducer, $P_{\mathrm{t}}$, can be also determined from the following relation by measuring the angular velocity of the transducer, $\Omega$ :

$$
P_{\mathrm{t}}=r_{\mathrm{t}} \Omega^{2}
$$

Table 1 Characteristics of the torsional transducer used in load testing.

\begin{tabular}{lc}
\hline Diameter $[\mathrm{mm}]$ & 30.0 \\
\hline Resonant frequency $[\mathrm{kHz}]$ & 24.3 \\
\hline Mechanical $Q$ & 130 \\
\hline Mechanical resistance $[\mathrm{Nms}]$ & 0.041 \\
\hline Damped capacitance $[\mathrm{nF}]$ & 3.21 \\
\hline Torque factor $[\mathrm{Nm} / \mathrm{V}]$ & 0.015 \\
\hline Dielectric loss $[\Omega]$ & $7.6 \times 10^{5}$ \\
\hline
\end{tabular}

The vibration characteristics used in the experiment is shown in Table 1 . The $(3,0)$ mode radiation plate of $100 \mathrm{~mm} \times 50 \mathrm{~mm}$ was used in load testing. Figure 11 shows the measured output power from the transducer and the efficiency. The applied voltage to the transducer remained constant at $50 \mathrm{~V}_{\mathrm{rms}}$, while the immersed depth of the radiation plate 


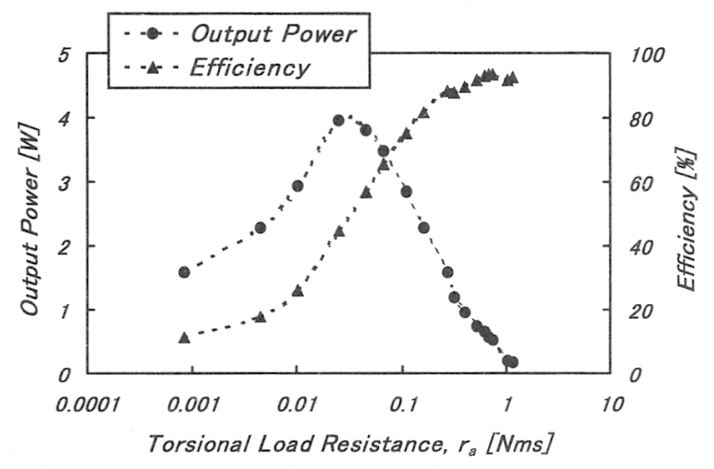

Fig. 11 Measured output power and efficiency as a function of the load resistance.

(load resistance) varied. The load resistance, which gives the maximum output power, is approximately identical to the mechanical resistance of the transducer, and this can be explained from the maximum power-transfer theorem. The efficiency of the transducer over $90 \%$ was measured at high load resistance. Load testing of the torsional transducer with high load resistance was effectively conducted by using the radiation plate designed by our method.

\section{SUMMARY}

The design method of the radiation plate with high load resistance for load testing of the torsional transducer has been studied. The analytic expression for calculating the torsional load impedance of the radiation plate was obtained and the validity was confirmed in the experiments. The method for designing the large radiation plate with high load resistance has been investigated and load testing of the torsional transducer was effectively conducted. The authors wish to investigate several important problems such as the influence of the radiation from the side and bottom faces of the radiation plate and generalizing the design method of the radiation plate for the driving frequency.

\section{ACKNOWLEDGEMENTS}

The authors would like to thank the members of the machine shop, Precision and Intelligence Laboratory, Tokyo Institute of Technology, for their assistance in fabricating the experimental equipment, and also thank Mr. James R. Friend, from the University of Missouri-Rolla, for helpful suggestions and observations. This work was partially supported by a Grant-in-Aid for COE Research from the Ministry of Education, Science, Sports and Culture.

\section{REFERENCES}

1) S. Nemoto and E. Mori, "Bolt-clamped electrostrictive torsional vibrator," J. Acoust. Soc. Jpn. (J) 28, 117-126 (1972) (in Japanese).

2) K. Adachi, Y. Konno, and S. Masaki, "Development of bolt-clamped Langevin-type transducer with high mechanical quality factor for excitation of large torsional vibration,” Jpn. J. Appl. Phys. 33, 1182-1188 (1994)

3) Y. Koike, M. Kihara, J. Satonobu, K. Nakamura, and S. Ueha, "A peripherally bolted torsional Langevin vibrator with large diameter," J. Acoust. Soc. Jpn. (E) 18, 239-246 (1997).

4) E. Mori, S. Ueha, Y. Tsuda, S. Kaneko, K. Okada, K. Ohbayashi, and K. Ohya, "Load test of bolt-clamped Langevin type vibrator," Tech. Rep. IEICE Jpn. US81-37, 17-24 (1981) (in Japanese).

5) E. Mori, S. Ueha, Y. Tsuda, S. Kaneko, K. Okada, M. Masuda, and K. Ohoya, "Load test of bolt-clamped Langevin type vibrator,” Jpn. J. Appl. Phys. 23, Suppl. 23-1, 209-211 (1984).

6) S. Ueha, M. Kuribayashi, Y. Tsuda, E. Mori, and Y. Hashimoto, "An ultrasonic power meter," J. Acoust. Soc. Am. 79, 985-989 (1986).

7) S. Kaneko, Y. Ootuki, S. Nomoto, and H. Yamamori, "Load characteristics of torsional vibrator," Proc. Autumn Meet. Acoust. Soc. Jpn. 2-1-10, 977-978 (1993) (in Japanese).

8) M. Gakumazawa, M. Shimada, S. Kaneko, and S. Nomoto, "Characteristics of torsional vibrator," Proc. Spring Meet. Acoust. Soc. Jpn. 1-2-19, 793-794 (1993) (in Japanese).

9) O. C. Zienkiewicz and R. L. Taylor, The Finite Element Method, 4th ed., Vol. 2 (McGraw-Hill Book Company Europe, U. K., 1991).

10) Ultrasonic Engineering (Corona Publishing Co., Ltd., Tokyo, 1993) (in Japanese).

11) J. Satonobu, F. Magane, Y. Koike, K. Nakamura, and S. Ueha, "Development of the torque accumulation method for a torsional vibration system," IEEE Trans. Ultrason. Ferroelectr. Freq. Control 44, 1348 1354 (1997).

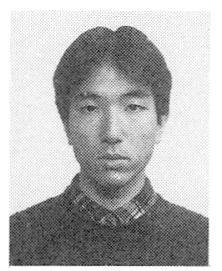

Masahiko Takakusaki was born in Kanagawa prefecture, Japan, on March 22, 1974. He received the B. Eng. degree from the Tokyo Institute of Technology, Tokyo, Japan in 1996. He is currently studying for the M. Eng. degree at the Tokyo Institute of Technology. His research interest has been in high power applications of ultrasonics. Mr. Takakusaki is a member of the Acoustical Society of Japan. 


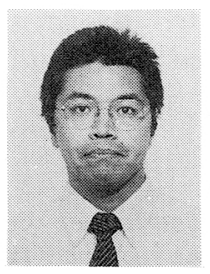

Jun Satonobu was born in Hiroshima prefecture, Japan, on August 1, 1964. He received the B. Eng. and the M. Eng. degree from the Tokyo Institute of Technology, Tokyo, Japan in 1987 and 1989, respectively. He is currently studying for the D. Eng. degree at the Tokyo Institute of Technology. His research interest has been in ultrasonic motors and actuators and in high power applications of ultrasonics. Mr. Satonobu is a member of the Acoustical Society of Japan.

Yoshikazu Koike was born in Niigata Prefecture, Japan, on November 29, 1965. He received the B. Eng. and the M. Eng. degrees from the Tokyo Institute of Technology, Tokyo, Japan, in 1989 and 1991, respectively. He has been a research associate of the Precision and Intelligence Laboratory, Tokyo Institute of Technology, since 1993. He has received the Awaya Kiyoshi Award for encouragement of research from the Acoustical Society of Japan in 1996. Mr. Koike is a member of the Acoustical Society of Japan and the Institute of Electronics, Information and Communication Engineers.

Kentaro Nakamura was born in Tokyo, Japan, on July 3, 1963. He received the B. Eng., the M. Eng., and the D. Eng. degrees from the Tokyo Institute of Technology, Tokyo, Japan, in 1987, 1989, and 1992, respectively. $\mathrm{He}$ has been an Associate Professor of the Precision and Intelligence Laboratory, Tokyo Institute of Technology, since
1996. His field of research is the application of ultrasonics and the measurement of vibration and sound using optical methods. He has received the Awaya Kiyoshi Award for encouragement of research from the Acoustical Society of Japan in 1996. Dr. Nakamura is a member of the Acoustical Society of Japan, the Japan Society of Applied Physics, the Institute of Electrical Engineers of Japan, and the Institute of Electronics, Information and Communication Engineers.

Sadayuki Ueha was born in Kyoto Prefecture, Japan, on February 28, 1943. He received the B. Eng. Degree in electronic engineering from the Nagoya Institute of Technology in 1965 and the M. Eng. Degree in 1967, and the D. Eng. Degree in 1970, both in electric engineering, from the Tokyo Institute of Technology. He currently conducts research in high power ultrasonics. He has been a Professor of the Precision and Intelligence Laboratory, Tokyo Institute of Technology since 1992. He is a chairman of steering committee member of the World Congress on Ultrasonics and served as the secretariat of WCU97. He received the Best Paper Award from The Japan Society of Applied Physics in 1975 and from the Acoustical Society of Japan in 1980, respectively. Dr. Ueha is a member of the Japan Society of Applied Physics, the Acoustical Society of Japan, the Institute of Electronics, Information and Communication Engineers, the Japan Society of Ultrasonics in Medicine and the Acoustical Society of America. Currently, he is a president of the Acoustical Society of Japan and a member of the editorial board of the journal "Ultrasonics." 